\title{
Well-posedness for parametric generalized vector quasivariational inequality problems of the Minty type
}

\section{Nguyen Van Hung ${ }^{*}$}

"Correspondence:

ngvhungdhdt@yahoo.com Department of Mathematics, Dong Thap University, 783 Pham Huu Lau Street, Ward 6, Cao Lanh City, Vietnam

\begin{abstract}
In this paper, we introduce the concepts of well-posedness, and of well-posedness in the generalized sense for parametric generalized vector quasivariational inequality problems of the Minty type. The necessary and sufficient conditions for the various kinds of well-posedness of these problems are obtained. Our results are different from some main results in the literature and extend them.

MSC: $90 \mathrm{C} 31 ; 49 J 53 ; 49 J 40 ; 49 J 45$

Keywords: parametric generalized vector quasivariational inequality problems of the Minty type; well-posed; well-posedness in the generalized sense; upper semicontinuity
\end{abstract}

\section{Introduction and preliminaries}

A vector variational inequality in a finite-dimensional Euclidean space was introduced first by Giannessi [1]. Later, this problem has been extended and studied by many authors in abstract spaces; see [2-6]. Moreover, vector variational inequality problems have many important applications in vector optimization problems [7-9], vector equilibria problems $[10,11]$, and variational relation problems $[12,13]$.

The concept of well-posedness for unconstrained scalar optimization problems was first introduced and studied by Tykhonov [8], which has become known as Tykhonov wellposedness. In 1966, Levitin and Polyak [14] introduced the concept of well-posedness for constrained scalar optimization problems. With the development of the theory about optimization problems, the concept of well-posedness has been generalized to several related problems, as vector optimization problems, see [15-20], variational inequality problems, see [15, 21-23], equilibria problems, see [24-33] and the references therein. Recently, Fang and Huang [22] studied the well-posedness for a vector variational inequality of the Minty type and the Stampacchia type. Very recently, Lalitha and Bhatia [23] also studied a quasivariational inequality problem of the Minty type, and the well-posedness for this problem was obtained.

Motivated and inspired by the work mentioned, in this paper, we also study the parametric generalized vector quasivariational inequality problems. However, we only study the well-posedness for generalized vector quasivariational inequality problems of the Minty type. The well-posedness for generalized vector quasivariational inequality problems of the Stampacchia type is the same as the Minty type. Let $X, Y, \Gamma, \Lambda$ be metric spaces and

(อ2014 Hung; licensee Springer. This is an Open Access article distributed under the terms of the Creative Commons Attribution License (http://creativecommons.org/licenses/by/2.0), which permits unrestricted use, distribution, and reproduction in any medium, provided the original work is properly cited. 
$C \subset Y$ be a closed, convex, and pointed cone with int $C \neq \emptyset$. The cone $C$ induces a partial ordering in $Y$ defined by

$$
\begin{aligned}
& y<x \quad \Leftrightarrow \quad y-x \in-\operatorname{int} C, \quad \forall x, y \in Y, \\
& y \nless x \quad \Leftrightarrow \quad y-x \notin-\operatorname{int} C, \quad \forall x, y \in Y,
\end{aligned}
$$

where int $C$ denotes the interior of $C$.

Let $L(X, Y)$ be the space of all linear continuous operators from $X$ into $Y$, and $A \subset X$ be a nonempty subset. Let $K_{1}: A \times \Gamma \rightarrow 2^{A}, K_{2}: A \times \Gamma \rightarrow 2^{A}$, and $T: A \times \Gamma \rightarrow 2^{L(X, Y)}$ be set-valued mappings. Let $Q: L(X, Y) \rightarrow L(X, Y), \eta: A \times A \times \Lambda \rightarrow A$ be continuous singlevalued mappings. We denote by $\langle z, x\rangle$ the value of a linear operator $z \in L(X ; Y)$ at $x \in X$, and we always assume that $\langle\cdot, \cdot \cdot\rangle$ is continuous.

Now we adopt the following notations (see $[10,12,13])$. For subsets $M$ and $N$ under consideration we adopt the notations

$$
\begin{array}{ll}
(u, v) \mathrm{w} M \times N \quad \text { means } \forall u \in M, \exists v \in N, & \text { means } \exists v \in N, \forall u \in M, \\
(u, v) \mathrm{m} M \times N \quad \text { means } \forall u \in M, \forall v \in N, \\
(u, v) \mathrm{s} M \times N \quad \text { means } \exists u \in M, \forall v \in N \text { and similarly for } \bar{m}, \bar{s} .
\end{array}
$$

where $\mathrm{w}, \mathrm{m}$, and $\mathrm{s}$ are used for weak, middle, and strong, respectively, kinds of considered problems. Let $\alpha \in\{\mathrm{w}, \mathrm{m}, \mathrm{s}\}, \bar{\alpha} \in\{\bar{w}, \bar{m}, \bar{s}\}$, and, for $\gamma \in \Gamma, \lambda \in \Lambda$. We consider the following parametric generalized vector quasivariational inequality problems of the Minty type (in short: (MQVIP $\left.{ }^{\gamma \lambda}\right)$ ).

(MQVIP $\left.{ }^{\gamma \lambda}\right)$ Find $\bar{x} \in K_{1}(\bar{x}, \gamma)$ such that $(y, z) \alpha K_{2}(\bar{x}, \gamma) \times T(y, \gamma)$ satisfies

$$
\langle Q(z), \eta(y, \bar{x}, \lambda)\rangle \nless 0 .
$$

Denote by (MQVIP) the family $\left\{\left(\mathrm{MQVIP}^{\gamma \lambda}\right):(\gamma, \lambda) \in \Gamma \times \Lambda\right\}$. For each $\gamma \in \Gamma, \lambda \in \Lambda$, and let $E(\gamma):=\left\{x \in A: x \in K_{1}(x, \gamma)\right\}$. We denote by $\Psi_{\alpha}(\gamma, \lambda)$ the solution sets of (MQVIP ${ }^{\gamma \lambda}$ ).

Throughout the article, we assume that $\Psi_{\alpha}(\gamma, \lambda) \neq \emptyset$ for each $(\gamma, \lambda)$ in the neighborhoods $\left(\gamma_{0}, \lambda_{0}\right) \in \Gamma \times \Lambda$.

Next, we recall some basic definitions and some of their properties.

Definition 1.1 ([34,35]) Let $X$ and $Z$ be two topological vector spaces and let $G: X \rightarrow 2^{Z}$ be a multifunction.

(i) $G$ is said to be lower semicontinuous (lsc) at $x_{0}$ if $G\left(x_{0}\right) \cap U \neq \emptyset$ for each open set $U \subseteq Z$ implies the existence of a neighborhood $V$ of $x_{0}$ such that $G(x) \cap U \neq \emptyset$, $\forall x \in V$.

(ii) $G$ is said to be upper semicontinuous (usc) at $x_{0}$ if for each open set $U \supseteq G\left(x_{0}\right)$, there is a neighborhood $V$ of $x_{0}$ such that $U \supseteq G(x), \forall x \in V$.

(iii) $G$ is said to be closed at $x_{0}$ if for each net $\left\{\left(x_{n}, y_{n}\right)\right\} \in \operatorname{graph} G:=\{(x, y) \mid y \in G(x)\}$, $\left(x_{n}, y_{n}\right) \rightarrow\left(x_{0}, y_{0}\right)$, it follows that $\left(x_{0}, y_{0}\right) \in \operatorname{graph} G$. 
Lemma $1.2([34,35])$ Let $X$ and $Z$ be two topological vector spaces and $G: X \rightarrow 2^{Z}$ be a multifunction.

(i) If $Z$ is compact and $G$ is closed at $x_{0}$, then $G$ is usc at $x_{0}$.

(ii) If $G$ is usc at $x_{0}$ and $G\left(x_{0}\right)$ is closed, then $G$ is closed at $x_{0}$.

The structure of this article is as follows. In the remaining part of this section, we recall definitions for later use. In Section 2, we introduce concepts of well-posedness, and well-posedness in the generalized sense for parametric generalized vector quasivariational inequality problems of the Minty type. Moreover, the necessary and sufficient conditions for the various kinds of well-posedness of these problems are obtained.

\section{Main results}

Definition 2.1 Let $\left\{\left(\gamma_{n}, \lambda_{n}\right)\right\} \subseteq \Gamma \times \Lambda$ converges to $\left(\gamma_{0}, \lambda_{0}\right)$. A sequence $\left\{x_{n}\right\} \subseteq A$ is said to be an approximating sequence for (MQVIP) corresponding to $\left\{\left(\gamma_{n}, \lambda_{n}\right)\right\}$, if

(i) $x_{n} \in K_{1}\left(x_{n}, \lambda_{n}\right), \forall n$;

(ii) there exists a sequence $\left\{\varepsilon_{n}\right\} \in \operatorname{int} C$ that converges to 0 such that

$$
(y, z) \alpha K_{2}\left(x_{n}, \gamma_{n}\right) \times T\left(y, \gamma_{n}\right) \quad \text { satisfies } \quad\left\langle Q(z), \eta\left(y, x_{n}, \lambda_{n}\right)\right\rangle+\varepsilon_{n} \nless 0 .
$$

Definition 2.2 The problem (MQVIP) is said to be well-posed at $\left(\gamma_{0}, \lambda_{0}\right)$ if

(i) the problem (MQVIP) has a unique solution $x_{0}$, i.e., $\Psi_{\alpha}\left(\gamma_{0}, \lambda_{0}\right)=\left\{x_{0}\right\}$;

(ii) for any sequence $\left\{\left(\gamma_{n}, \lambda_{n}\right)\right\} \subseteq \Gamma \times \Lambda$ converges to $\left(\gamma_{0}, \lambda_{0}\right)$, every approximating sequence $\left\{x_{n}\right\}$ for (MQVIP) corresponding to $\left\{\left(\gamma_{n}, \lambda_{n}\right)\right\}$ converges to $x_{0}$.

Definition 2.3 The problem (MQVIP) is said to be well-posed in the generalized sense at $\left(\gamma_{0}, \lambda_{0}\right)$ if

(i) the solution set $\Psi_{\alpha}\left(\gamma_{0}, \lambda_{0}\right)$ of (MQVIP) is nonempty;

(ii) for any sequence $\left\{\left(\gamma_{n}, \lambda_{n}\right)\right\} \subseteq \Gamma \times \Lambda$ that converges to $\left(\gamma_{0}, \lambda_{0}\right)$, every approximating sequence $\left\{x_{n}\right\}$ for (MQVIP) corresponding to $\left\{\left(\gamma_{n}, \lambda_{n}\right)\right\}$ has a subsequence which converges to some point of $\Psi_{\alpha}\left(\gamma_{0}, \lambda_{0}\right)$.

For $\gamma \in \Gamma, \lambda \in \Lambda$, and $\varepsilon \in \operatorname{int} C$, we denote the approximate solution set of (MQVIP) by $\Omega(\gamma, \lambda, \varepsilon)$

$$
\Omega(\gamma, \lambda, \varepsilon):=\left\{x \in K_{1}(x, \gamma) \mid(y, z) \alpha K_{2}(x, \gamma) \times T(y, \gamma):\{Q(z), \eta(y, x, \lambda)\rangle+\varepsilon \nless 0\right\} .
$$

\section{Remark 2.4}

(i) In the special case, where $A=B, X=Y, \Gamma=\Lambda, K_{1}(x, \gamma)=K_{2}(x, \gamma)=A$, $\eta(y, x, \lambda)=y-x$, and $Q$ is an identity map, let $T: A \times \Gamma \rightarrow L(X, Y)$ be a single-valued mapping, then the problem (MQVIP ${ }^{\gamma \lambda}$ ) reduces to the problem $\left(\mathrm{MVVI}^{\lambda}\right.$ ) studied in [22].

(ii) In the special case as in Remark 2.4(i), then Definitions 2.1, 2.2, and 2.3 reduce to Definitions 2.2, 2.5, and 2.6, respectively, of Fang and Huang in [22].

(iii) Well-posedness for vector problems has been defined in different ways. In this paper, we denote $\varepsilon \in \operatorname{int} C$ instead of $\epsilon e$, with $\epsilon$ being positive numbers and $e \in \operatorname{int} C$, i.e., only a fixed direction $e$ is allowed (see $[24,28]$ ). 
Remark 2.5 ([36]) Let $X$ and $Z$ be two metric spaces and $G: X \rightarrow 2^{Z}$ be a multifunction. If $G\left(x_{0}\right)$ is compact, then $G$ is usc at $x_{0}$ if and only if for any sequence $\left\{x_{n}\right\}$ that converges to $x_{0}$ and for any sequence $\left\{y_{n}\right\} \subseteq G\left(x_{n}\right)$, there is a subsequence $\left\{y_{n_{k}}\right\}$ of $\left\{y_{n}\right\}$ converging to some $y_{0} \in G\left(x_{0}\right)$. If, in addition, $G\left(x_{0}\right)=\left\{y_{0}\right\}$ is a singleton, then the above limit point $y$ must be $y_{0}$ and the whole $\left\{y_{n}\right\}$ converges to $y_{0}$.

The following theorem gives sufficient conditions for the well-posedness and the wellposedness in the generalized sense for (MQVIP).

Theorem 2.6 Assume for problem (MQVIP) that

(i) $E$ is usc at $\gamma_{0}$ and $E\left(\gamma_{0}\right)$ is a compact set;

(ii) in $K_{1}(A, \Gamma) \times\left\{\gamma_{0}\right\}, K_{2}$ is lsc;

(iii) in $K_{2}\left(K_{1}(A, \Gamma), \Gamma\right) \times\left\{\gamma_{0}\right\}, T$ is usc and compact-valued if $\alpha=\mathrm{w}($ or $\alpha=\mathrm{m})$, and lsc if $\alpha=\mathrm{s}$.

Then (MQVIP) is well-posed in the generalized sense at $\left(\gamma_{0}, \lambda_{0}\right)$. Moreover, if $\Psi_{\alpha}\left(\gamma_{0}, \lambda_{0}\right)$ is a singleton, then this problem is well-posed at $\left(\gamma_{0}, \lambda_{0}\right)$.

Proof Since $\alpha=\{\mathrm{w}, \mathrm{m}, \mathrm{s}\}$, we have in fact three cases. However, the proof techniques are similar. We consider only the case $\alpha=\mathrm{s}$. We first prove that $\Omega_{s}$ is upper semicontinuous at $\left(\gamma_{0}, \lambda_{0}, 0\right)$. Indeed, we suppose to the contrary the existence of an open subset $V$ of $\Omega_{s}\left(\gamma_{0}, \lambda_{0}, 0\right)$ such that for all $\left\{\left(\gamma_{n}, \lambda_{n}, \varepsilon_{n}\right)\right\} \subseteq \Gamma \times \Lambda \times C$ it converges to $\left\{\left(\gamma_{0}, \lambda_{0}, 0\right)\right\}$, that is, $x_{n} \in \Omega_{s}\left(\gamma_{n}, \lambda_{n}, \varepsilon_{n}\right), x_{n} \notin V$, for all $n$. Since $E$ is usc and is compact-valued at $\gamma_{0}$, we can assume that $x_{n}$ tends to $x_{0}$ for some $x_{0} \in E\left(\gamma_{0}\right)$. If $x_{0} \notin \Omega_{s}\left(\gamma_{0}, \lambda_{0}, 0\right)=\Psi\left(\gamma_{0}, \lambda_{0}\right), \exists y_{0} \in$ $K_{2}\left(x_{0}, \gamma_{0}\right), \exists z_{0} \in T\left(y_{0}, \gamma_{0}\right)$ such that

$$
\left\langle Q\left(z_{0}\right), \eta\left(y_{0}, x_{0}, \lambda_{0}\right)\right\rangle<0 \text {. }
$$

By the lower semicontinuity of $K_{2}, T$ at $\left(x_{0}, \gamma_{0}\right)$ and $\left(y_{0}, \gamma_{0}\right), \forall y_{0} \in K_{2}\left(x_{0}, \gamma_{0}\right), \forall z_{0} \in$ $T\left(y_{0}, \gamma_{0}\right)$ there exists $y_{n} \in K_{2}\left(x_{n}, \gamma_{n}\right), z_{n} \in T\left(y_{n}, \gamma_{n}\right)$ such that $y_{n} \rightarrow y_{0}, z_{n} \rightarrow z_{0}$. Since $x_{n} \in \Omega\left(\gamma_{n}, \lambda_{n}, \varepsilon_{n}\right)$, we have

$$
\left\langle Q\left(z_{n}\right), \eta\left(y_{n}, x_{n}, \lambda_{n}\right)\right\rangle+\varepsilon_{n} \nless 0 .
$$

Let id : $C \rightarrow C$ be an identity map, by the continuity of $\eta, Q$, and $\langle\cdot, \cdot\rangle$, it follows that $\langle\cdot, \cdot\rangle+$ id is continuous (where id is continuous). So (2.1) implies

$$
\left\langle Q\left(z_{0}\right), \eta\left(y_{0}, x_{0}, \lambda_{0}\right)\right\rangle \nless 0,
$$

which is impossible. Hence, $x_{0}$ belongs to $\Omega_{s}\left(\gamma_{0}, \lambda_{0}, 0\right) \subseteq V$, which is again a contradiction, since $x_{n} \notin V$, for all $n$. Therefore, $\Omega_{s}$ is usc at $\left(\gamma_{0}, \lambda_{0}, 0\right)$.

Now we prove that $\Omega_{s}\left(\gamma_{0}, \lambda_{0}, 0\right)$ is compact, by checking its closedness. Indeed, let $x_{n} \in$ $\Omega_{s}\left(\gamma_{0}, \lambda_{0}, 0\right), x_{n} \rightarrow x_{0}$. This proof is similar to above and so we have $x_{0} \in \Omega_{s}\left(\gamma_{0}, \lambda_{0}, 0\right)$ and hence $\Omega_{s}\left(\gamma_{0}, \lambda_{0}, 0\right)$ is compact. By Remark 2.5, we complete the proof.

The following example shows that the upper semicontinuity and compactness of $E$ are essential. 
Example 2.7 Let $A=B=X=Y=\mathbb{R}, \Gamma=\Lambda=[0,1], C=\mathbb{R}_{+}, \gamma_{0}=0$, $Q$ be an identity map, $K_{1}, K_{2}: A \times \Gamma \rightarrow 2^{A}, T: A \times \Gamma \rightarrow 2^{L(X, Y)}$, and $\eta: A \times A \times \Gamma \rightarrow A$ be defined by

$$
\begin{aligned}
& K_{1}(x, \gamma)=\left(-\gamma-\frac{1}{2}, \gamma\right], \\
& \eta(y, x, \gamma)=\left\{\gamma^{2}+\gamma+2+\varepsilon\right\}, \\
& T(y, \gamma)=\left\{\frac{1}{2^{\gamma+2}}\right\}, \\
& K_{2}(x, \gamma)=\left[0,2^{\gamma^{2}+1}\right] .
\end{aligned}
$$

Then we have $E(0)=\left(-\frac{1}{2}, 0\right]$ and $E(\gamma)=\left(-\gamma-\frac{1}{2}, \gamma\right], \forall \gamma \in(0,1]$. We show that assumptions (ii) and (iii) of Theorem 2.6 are fulfilled. But the family $\left\{\left(\operatorname{MQVIP}^{\gamma \lambda}\right):(\gamma, \lambda) \in \Gamma \times \Lambda\right\}$ is not well-posed in the generalized sense at $(0,0)$. The reason is that $E$ is not usc at 0 and $E(0)$ is not compact. In fact

$$
\Omega_{\alpha}(\gamma, \lambda, \varepsilon)= \begin{cases}\left(-\frac{1}{2}, 0\right], & \text { if } \gamma=0, \\ \left(-\gamma-\frac{1}{2}, \gamma\right], & \text { if } \gamma \in(0,1] .\end{cases}
$$

The following example shows that the lower semicontinuity of $K_{2}$ is essential.

Example 2.8 Let $A=B=[-1,1], X=Y=\mathbb{R}, \Gamma=\Lambda=[0,1], C=\mathbb{R}_{+}, \gamma_{0}=0, Q$ be an identity map, $K_{1}, K_{2}: A \times \Gamma \rightarrow 2^{A}, T: A \times \Gamma \rightarrow 2^{L(X, Y)}$, and $\eta: A \times A \times \Gamma \rightarrow A$ be defined by

$$
\begin{aligned}
& K_{1}(x, \gamma)=[0,1], \\
& \eta(y, x, \gamma)=\{x+y-\varepsilon\}, \\
& T(y, \gamma)=\{1\}, \\
& K_{2}(x, \gamma)= \begin{cases}\{-1,0,1\}, & \text { if } \gamma=0, \\
\{0,1\}, & \text { otherwise. }\end{cases}
\end{aligned}
$$

We have $E(\gamma)=[0,1], \forall \gamma \in[0,1]$. Hence $E$ is usc at 0 and $E(0)$ is compact and the conditions (ii) and (iii) of Theorem 2.6 are easily seen to be fulfilled. But the family $\left\{\left(\mathrm{MQVIP}^{\gamma \lambda}\right):(\gamma, \lambda) \in \Gamma \times \Lambda\right\}$ is not well-posed in the generalized sense at $(0,0)$. The reason is that $K_{2}$ is not lower semicontinuous at $(x, 0)$. In fact

$$
\Omega_{\alpha}(\gamma, \lambda, \varepsilon)= \begin{cases}\{1\}, & \text { if } \gamma=0, \\ {[0,1],} & \text { if } \gamma \in(0,1] .\end{cases}
$$

Theorem 2.9 Assume for problem (MQVIP) the assumptions (ii) and (iii) as in Theorem 2.6 and replace (i) by (i'):

(i') $A$ is compact, $K_{1}$ is closed in $A \times\left\{\gamma_{0}\right\}$.

Then (MQVIP) is well-posed in the generalized sense at $\left(\gamma_{0}, \lambda_{0}\right)$. Moreover, if $\Psi_{\alpha}\left(\gamma_{0}, \lambda_{0}\right)$ is a singleton, then this problem is well-posed at $\left(\gamma_{0}, \lambda_{0}\right)$. 
Proof We omit the proof since the technique is similar to that for Theorem 2.6 with suitable modifications.

The following example shows that the compactness of $A$ cannot be dropped.

Example 2.10 Let $A=B=X=Y=(-\infty,+\infty), \Gamma=\Lambda=[0,+\infty), C=[0,+\infty), \gamma_{0}=0, H$ be the identity map, $K_{1}, K_{2}: A \times \Gamma \rightarrow 2^{A}, T: A \times \Gamma \rightarrow 2^{B}$, and $\eta: A \times A \times \Gamma \rightarrow A$ be defined by

$$
\begin{aligned}
& K_{1}(x, \gamma)= \begin{cases}\{0\}, & \text { if } \gamma=0, \\
\left\{2^{1+\gamma}\right\}, & \text { if } \gamma \neq 0,\end{cases} \\
& K_{2}(x, \gamma)=[0,1], \\
& \eta(y, x, \lambda)=\left\{\frac{1}{\left.2^{\gamma+\gamma^{2}}-\varepsilon 3^{\cos ^{2}(\gamma)+\gamma+1}\right\},}\right. \\
& T(y, \gamma)=\left\{\frac{1}{\left.3^{\cos ^{2}(\gamma)+\gamma+1}\right\} .}\right.
\end{aligned}
$$

We see that $K_{1}$ is closed at $(x, 0)$, the assumptions (ii) and (iii) of Theorem 2.9 are satisfied. But the family $\left\{\left(\right.\right.$ MQVIP $\left.\left.^{\gamma \lambda}\right):(\gamma, \lambda) \in \Gamma \times \Lambda\right\}$ is not well-posed in the generalized sense at $(0,0)$. The reason is that $A$ is not compact. In fact, $\Omega_{\alpha}(0,0,0)=\{0\}$ and $\Omega_{\alpha}(\gamma, \lambda, \varepsilon)=\left\{2^{1+\gamma}\right\}$, $\forall \gamma \in(0,+\infty)$.

The following example shows that the closedness of $K_{1}$ is essential.

Example 2.11 Let $A=B=X=Y=[-3,3], \Gamma=\Lambda=[0,1], C=\mathbb{R}_{+}, \gamma_{0}=0, H$ be an identity map, $K_{1}, K_{2}: A \times \Gamma \rightarrow 2^{A}, T: A \times \Gamma \rightarrow 2^{B}$, and $\eta: A \times A \times \Gamma \rightarrow A$ be defined by

$$
\begin{aligned}
& K_{1}(x, \gamma)=(-3 \gamma, 3], \\
& K_{2}(x, \gamma)=[0,3], \\
& \eta(y, x, \gamma)=\left\{x^{2}-y x-\varepsilon\right\}, \\
& T(y, \gamma)=\{1\} .
\end{aligned}
$$

We show that $A$ is compact and the conditions (ii), (iii) of Theorem 2.9 are easily seen to be fulfilled. But the family $\left\{\left(\operatorname{MQVIP}^{\gamma \lambda}\right):(\gamma, \lambda) \in \Gamma \times \Lambda\right\}$ is not well-posed in the generalized sense at $(0,0)$. The reason is that $K_{1}$ is not closed at $(x, 0)$. In fact,

$$
\Omega_{\alpha}(\gamma, \lambda, \varepsilon)= \begin{cases}\{3\}, & \text { if } \gamma=0, \\ \{0,3\}, & \text { if } \gamma \neq 0 .\end{cases}
$$

The following example shows that all assumptions of Theorem 2.6 are satisfied.

Example 2.12 Let $X=Y=\mathbb{R}, A=B=[0,3], \Gamma=\Lambda=[0,1], C=\mathbb{R}_{+}, \gamma_{0}=0, H$ be an identity map, and let $K_{1}, K_{2}: A \times \Gamma \rightarrow 2^{A}, T: A \times \Gamma \rightarrow 2^{L(X, Y)}$, and $\eta: A \times A \times \Gamma \rightarrow A$ be defined 
by

$$
\begin{aligned}
& K_{1}(x, \gamma)=K_{2}(x, \gamma)=[0,1], \\
& \eta(y, x, \gamma)=\gamma^{2}+2 \gamma+1-\varepsilon, \\
& T(y, \gamma)=\{1\} .
\end{aligned}
$$

Then $E(\gamma)=[0,1], \forall \gamma \in[0,1]$. We see that all assumptions of Theorem 2.9 are satisfied. So, the family $\left\{\left(\mathrm{MQVIP}^{\gamma \lambda}\right):(\gamma, \lambda) \in \Gamma \times \Lambda\right\}$ is well-posed in the generalized sense at $(0,0)$. In fact, $\Omega(\gamma, \lambda, \varepsilon)=[0,1], \forall \gamma \in[0,1]$.

For $(\gamma, \lambda) \in \Gamma \times \Lambda, \varepsilon \in \operatorname{int} C$, and positive $\xi$, we define the following sets of approximate solutions of the family $\left\{\left(\mathrm{MQVIP}^{\gamma \lambda}\right):(\gamma, \lambda) \in \Gamma \times \Lambda\right\}$ :

$$
\begin{aligned}
& \Sigma_{\alpha}^{\gamma_{0} \lambda_{0}}(\xi, \varepsilon) \\
& \quad=\bigcup_{\gamma \in B\left(\gamma_{0}, \xi\right), \lambda \in B\left(\lambda_{0}, \xi\right)} \Omega_{\alpha}(\gamma, \lambda, \varepsilon) \\
& \quad=\bigcup_{\gamma \in B\left(\gamma_{0}, \xi\right), \lambda \in B\left(\lambda_{0}, \xi\right)}\left\{x \in K_{1}(x, \gamma) \mid(y, z) \alpha K_{2}(x, \gamma) \times T(y, \gamma):\langle Q(z), \eta(y, x, \lambda)\rangle+\varepsilon \nless 0\right\},
\end{aligned}
$$

where $B\left(\gamma_{0}, \xi\right)$ and $B\left(\lambda_{0}, \xi\right)$ are the closed balls centered at $\gamma_{0}$ and $\lambda_{0}$ with radius $\xi$.

Observe that, for every $(\gamma, \lambda) \in \Gamma \times \Lambda$,

(i) $\Sigma_{\alpha}^{\gamma_{0} \lambda_{0}}(0,0)=\Omega_{\alpha}\left(\gamma_{0}, \lambda_{0}, 0\right)=\Psi_{\alpha}\left(\gamma_{0}, \lambda_{0}\right)$;

(ii) $\Psi_{\alpha}\left(\gamma_{0}, \lambda_{0}\right) \subseteq \Omega_{\alpha}\left(\gamma_{0}, \lambda_{0}, \varepsilon\right) \subseteq \Sigma_{\alpha}^{\gamma_{0} \lambda_{0}}(\xi, \varepsilon)$.

Theorem 2.13 Assume $X$ is complete and the following conditions hold:

(i) $K_{1}$ is closed in $A \times\left\{\gamma_{0}\right\}$, and in $K_{1}(A, \Gamma) \times\left\{\gamma_{0}\right\}, K_{2}$ is lsc;

(ii) in $K_{2}\left(K_{1}(A, \Gamma), \Gamma\right) \times\left\{\gamma_{0}\right\}, T$ is usc and compact-valued if $\alpha=\mathrm{w}($ or $\alpha=\mathrm{m})$, and lsc if $\alpha=\mathrm{s}$.

Then (MQVIP) is well-posed at $\left(\gamma_{0}, \lambda_{0}\right)$ if and only if

$$
\Sigma_{\alpha}^{\gamma_{0} \lambda_{0}}(\xi, \varepsilon) \neq \emptyset, \quad \forall \xi>0, \varepsilon \in \operatorname{int} C \quad \text { and } \quad \operatorname{diam} \Sigma_{\alpha}^{\gamma_{0} \lambda_{0}}(\xi, \varepsilon) \rightarrow 0 \quad \text { as }(\xi, \varepsilon) \rightarrow(0,0)
$$

Proof Similar arguments can be applied to the three cases. We present only the proof for the case where $\alpha=$ s. If (MQVIP) is well-posed at $\left(\gamma_{0}, \lambda_{0}\right)$, then (MQVIP) has a unique solution $x_{0} \in \Psi_{s}\left(\gamma_{0}, \lambda_{0}\right)$ and hence $\Sigma_{s}^{\gamma_{0} \lambda_{0}}(\xi, \varepsilon) \neq \emptyset, \forall \xi>0, \varepsilon \in \operatorname{int} C$ as $\Psi_{s}\left(\gamma_{0}, \lambda_{0}\right) \subseteq \Sigma_{s}^{\gamma_{0} \lambda_{0}}(\xi, \varepsilon)$. If $\operatorname{diam} \Sigma_{s}^{\gamma_{0} \lambda_{0}}(\xi, \varepsilon) \nrightarrow 0$ as $(\xi, \varepsilon) \rightarrow(0,0)$, then there exist $q>0$ and $\xi_{n}>0, \varepsilon_{n} \in \operatorname{int} C$, such that $\varepsilon_{n} \rightarrow 0, \xi_{n} \rightarrow 0$, and

$$
\operatorname{diam} \Sigma_{s}^{\gamma_{0} \lambda_{0}}\left(\xi_{n}, \varepsilon_{n}\right)>q>0, \quad \forall n \in \mathbb{N}
$$

Then there exist $x_{n}^{1}, x_{n}^{2} \in \Sigma_{s}^{\gamma_{0} \lambda_{0}}\left(\xi_{n}, \varepsilon_{n}\right)$ such that $d\left(x_{n}^{1}, x_{n}^{2}\right)>\frac{q}{2}>0$. Hence there exist $\gamma_{n}^{1}, \gamma_{n}^{2} \in$ $B\left(\gamma_{0}, \xi_{n}\right)$, and $\lambda_{n}^{1}, \lambda_{n}^{2} \in B\left(\lambda_{0}, \xi_{n}\right)$ such that $\forall y \in K_{2}\left(x_{n}^{1}, \gamma_{n}^{1}\right), \forall z \in T\left(y, \gamma_{n}^{1}\right)$ satisfy

$$
\left\langle Q(z), \eta\left(y, x_{n}^{1}, \lambda_{n}^{1}\right)\right\rangle+\varepsilon_{n} \nless 0,
$$

and $\forall y \in K_{2}\left(x_{n}^{2}, \gamma_{n}^{2}\right), \forall z \in T\left(y, \gamma_{n}^{2}\right)$ satisfy

$$
\left\langle Q(z), \eta\left(y, x_{n}^{2}, \lambda_{n}^{2}\right)\right\rangle+\varepsilon_{n} \nless 0,
$$


i.e., $\left\{x_{n}^{1}\right\}$ and $\left\{x_{n}^{2}\right\}$ are approximating sequences for (MQVIP) corresponding to $\left\{\left(\gamma_{n}^{1}, \lambda_{n}^{1}\right)\right\}$ and $\left\{\left(\gamma_{n}^{2}, \lambda_{n}^{2}\right)\right\}$, respectively. Hence, the sequences $\left\{x_{n}^{1}\right\}$ and $\left\{x_{n}^{2}\right\}$ converges to the unique solution $x_{0}$ of (MQVIP $\left.{ }^{\gamma_{0} \lambda_{0}}\right)$, contradicting the fact that $d\left(x_{n}^{1}, x_{n}^{2}\right)>\frac{q}{2}>0, \forall n \in \mathbb{N}$.

Conversely, let $\left\{\gamma_{n}\right\} \rightarrow \gamma_{0}$ and $\left\{\lambda_{n}\right\} \rightarrow \lambda_{0}$, and $\left\{x_{n}\right\}$ be approximating sequences for (MQVIP) corresponding to $\left\{\gamma_{n}\right\}$ and $\left\{\lambda_{n}\right\}$. Then there is $\left\{\varepsilon_{n}\right\} \rightarrow 0$ such that $\forall y \in K_{2}\left(x_{n}, \gamma_{n}\right)$, $\forall z \in T\left(y, \gamma_{n}\right)$ satisfying

$$
\left\langle Q(z), \eta\left(y, x_{n}, \lambda_{n}\right)\right\rangle+\varepsilon_{n} \nless 0, \quad \forall n \in \mathbb{N} .
$$

This yields $x_{n} \in \Sigma_{s}^{\gamma_{0} \lambda_{0}}\left(\xi_{n}, \varepsilon_{n}\right)$ with $\left\{\xi_{n}\right\}=\max \left\{d\left(\gamma_{n}, \gamma_{0}\right), d\left(\lambda_{n}, \lambda_{0}\right)\right\} \rightarrow 0$, as $n \rightarrow+\infty$. Since $\operatorname{diam} \Sigma_{s}^{\gamma_{0} \lambda_{0}}\left(\xi_{n}, \varepsilon_{n}\right) \rightarrow 0$ as $\left(\xi_{n}, \varepsilon_{n}\right) \rightarrow(0,0)$, it follows that $\left\{x_{n}\right\}$ is Cauchy and converges to a point $x_{0}$. By the closedness of $K_{1}$ at $\left(x_{0}, \gamma_{0}\right), x_{0} \in K_{1}\left(x_{0}, \gamma_{0}\right)$.

Next, we verify that $x_{0} \in \Psi_{s}\left(\gamma_{0}, \lambda_{0}\right)$. Using the same argument as for Theorem 2.6, we deduce that $x_{0} \in \Psi_{s}\left(\gamma_{0}, \lambda_{0}\right)$.

Now we prove that (MQVIP $\left.{ }^{\gamma_{0} \lambda_{0}}\right)$ has a unique solution. If $\Psi_{s}\left(\gamma_{0}, \lambda_{0}\right)$ has two distinct solutions $x_{1}$ and $x_{2}$, it is not hard to see that $x_{1}, x_{2} \in \Sigma_{s}^{\gamma_{0} \lambda_{0}}(\xi, \varepsilon), \forall \xi>0, \varepsilon \in \operatorname{int} C$. It follows that

$$
0<d\left(x_{1}, x_{2}\right) \leq \Sigma_{s}^{\gamma_{0} \lambda_{0}}(\xi, \varepsilon) \rightarrow 0,
$$

which is impossible. Hence, (MQVIP) is well-posed at $\left(\gamma_{0}, \lambda_{0}\right)$.

The following example shows that the uniqueness of well-posed is essential.

Example 2.14 Let $X=Y=\mathbb{R}, A=B=[-1,1], \Gamma=\Lambda=[0,1], C=\mathbb{R}_{+}, \gamma_{0}=0, H$ be an identity map, and let $K_{1}, K_{2}: A \times \Gamma \rightarrow 2^{A}, T: A \times \Gamma \rightarrow 2^{L(X, Y)}$, and $\eta: A \times A \times \Gamma \rightarrow A$ be defined by

$$
\begin{aligned}
& K_{1}(x, \gamma)=K_{2}(x, \gamma)=[0,1], \\
& \eta(y, x, \gamma)=\gamma+1-\varepsilon, \\
& T(y, \gamma)=\{1\} .
\end{aligned}
$$

We show that the conditions (i) and (ii) of Theorem 2.13 are easily seen to be fulfilled and the family $\left\{\left(\operatorname{MQVIP}^{\gamma \lambda}\right):(\gamma, \lambda) \in \Gamma \times \Lambda\right\}$ is well-posed at $(0,0)$. But $\operatorname{diam} \Sigma_{\alpha}^{\gamma_{0} \lambda_{0}}(\xi, \varepsilon)=$ $[0,1] \nrightarrow 0$ as $(\xi, \varepsilon) \rightarrow(0,0)$.

The following example shows that all assumptions of Theorem 2.13 are satisfied.

Example 2.15 Let $A=B=X=Y=\mathbb{R}, \Gamma=\Lambda=[0,1], C=\mathbb{R}_{+}, \gamma_{0}=0, H$ be an identity map, and let $K_{1}, K_{2}: A \times \Gamma \rightarrow 2^{A}, T: A \times \Gamma \rightarrow 2^{L(X, Y)}$, and $\eta: A \times A \times \Gamma \rightarrow A$ be defined by

$$
\begin{aligned}
& K_{1}(x, \gamma)=[0,+\infty), \\
& \eta(y, x, \gamma)=y-x+\gamma, \\
& T(y, \gamma)=\{1\}, \\
& K_{2}(x, \gamma)=[0,1] .
\end{aligned}
$$


We show that the conditions (i) and (ii) of Theorem 2.13 are easily seen to be fulfilled and $\Psi_{\alpha}(0,0)=\{0\}$ and

$$
\Omega_{\alpha}(\gamma, \lambda, \varepsilon)= \begin{cases}{[0, \varepsilon],} & \text { if } \gamma=0 \\ {[0, \gamma+\varepsilon],} & \text { if } \gamma \in(0,1]\end{cases}
$$

and $\Sigma_{\alpha}^{\gamma_{0} \lambda_{0}}(\xi, \varepsilon)=[0, \varepsilon]$ and the family $\left\{\left(\operatorname{MQVIP}^{\gamma \lambda}\right):(\gamma, \lambda) \in \Gamma \times \Lambda\right\}$ is well-posed at $(0,0)$, and $\operatorname{diam} \Sigma_{\alpha}^{\gamma_{0} \lambda_{0}}(\xi, \varepsilon) \rightarrow 0$ as $(\xi, \varepsilon) \rightarrow(0,0)$.

Next, we consider the following notions of measures of noncompactness.

Definition 2.16 ([37, 38]) Let $X$ is complete. The Kuratowski measure of the set $A \subseteq X$ is defined by

$$
\zeta(A)=\inf \left\{\vartheta>0 \mid A \subseteq \bigcup_{i=1}^{n} L_{i}, \operatorname{diam} L_{i}<\vartheta, i=1,2, \ldots, n, \text { for some } n \in \mathbb{N}\right\} .
$$

Definition $2.17([37,38]) A, B$ be nonempty subsets of $X$. The Hausdorff metric $H(\cdot, \cdot)$ between $A$ and $B$ is defined by

$$
H(A, B)=\max \left\{H^{*}(A, B), H^{*}(B, A)\right\},
$$

where $H^{*}(A, B)=\sup _{a \in A} d(a, B)$ with $d(a, B)=\inf _{b \in B}\|a-b\|$.

By the definitions of $\zeta$ and $H$, we have

$$
\zeta(A) \leq \zeta(B)+2 H(A, B)
$$

for every all bounded sets $A$ and $B$.

Remark 2.18 $([39,40])$ The function $\zeta$ is a regular measure of noncompactness defined by $\zeta: 2^{X} \rightarrow[0,+\infty]$ that satisfies the following conditions:

(i) $\zeta(D)=+\infty$ if and only if the set $D$ is unbounded;

(ii) $\zeta(D)=\zeta(\operatorname{cl}(D))$;

(iii) from $\zeta(D)=0$ it follows that $D$ is a totally bounded set;

(iv) from $P \subseteq Q$ it follows that $\zeta(P) \leq \zeta(Q)$;

(v) if $X$ is a complete space, and if $\left\{B_{n}\right\}$ is a sequence of closed subsets of $X$ such that $B_{n+1} \subseteq B_{n}$ for each $n \in \mathbb{N}$ and $\lim _{n \rightarrow+\infty} \zeta\left(B_{n}\right)=0$, then $M=\bigcap_{n \in N} B_{n}$ is a nonempty compact set and $\lim _{n \rightarrow+\infty} H\left(B_{n}, M\right)=0$, where $H$ is a Hausdorff metric.

Lemma 2.19 Assume we have problem (MQVIP). Let $\Gamma, \Lambda$ be finite dimensional and the following conditions hold:

(i) $K_{1}$ is closed in $A \times\left\{\gamma_{0}\right\}$, and in $K_{1}(A, \Gamma) \times\left\{\gamma_{0}\right\}, K_{2}$ is lsc;

(ii) in $K_{2}\left(K_{1}(A, \Gamma), \Gamma\right) \times\left\{\gamma_{0}\right\}, T$ is usc and compact-valued if $\alpha=\mathrm{w}$ (or $\left.\alpha=\mathrm{m}\right)$, and lsc if $\alpha=\mathrm{s}$.

Then $\Sigma_{\alpha}^{\gamma_{\alpha} \lambda_{0}}(\xi, \varepsilon)$ is closed, for all $\xi>0, \varepsilon \in \operatorname{int} C$.

Proof Similar arguments can be applied in the three cases. We present only the proof for the case where $\alpha=\mathrm{s}$. We let $x_{n} \in \Sigma_{s}^{\gamma_{0} \lambda_{0}}(\xi, \varepsilon)$ such that $x_{n} \rightarrow x$. Hence, for all $n \in \mathbb{N}$, there 
exist $\gamma_{n} \in B\left(\gamma_{0}, \xi\right)$ and $\lambda_{n} \in B\left(\lambda_{0}, \xi\right)$ and $\forall y \in K_{2}\left(x_{n}, \gamma_{n}\right), \forall z \in T\left(y, \gamma_{n}\right)$ such that

$$
\left\langle Q(z), \eta\left(y, x_{n}, \lambda_{n}\right)\right\rangle+\varepsilon \nless 0, \quad \forall n \in \mathbb{N} .
$$

Since $B\left(\gamma_{0}, \xi\right)$ and $B\left(\lambda_{0}, \xi\right)$ are compact, we can assume that $\left\{\gamma_{n}\right\} \rightarrow \gamma \in B\left(\gamma_{0}, \xi\right)$ and $\left\{\lambda_{n}\right\} \rightarrow \lambda \in B\left(\lambda_{0}, \xi\right)$. By the closedness of $K_{1}$ at $(x, \gamma)$, we find that $x \in K_{1}(x, \gamma)$. We show that $\forall y \in K_{2}(x, \gamma), \forall z \in T(y, \gamma)$ such that

$$
\langle Q(z), \eta(y, x, \lambda)\rangle+\varepsilon \nless 0,
$$

i.e., $x \in \Sigma_{s}^{\gamma_{0} \lambda_{0}}(\xi, \varepsilon)$. Indeed, if $x \notin \Sigma_{s}^{\gamma_{0} \lambda_{0}}(\xi, \varepsilon)$, then $\exists y \in K_{2}(x, \gamma), \exists z \in T(y, \gamma)$ such that

$$
\langle Q(z), \eta(y, x, \lambda)\rangle+\varepsilon<0
$$

By the lower semicontinuity of $K_{2}$ and $T$, there exist $y_{n} \in K_{2}\left(x_{n}, \gamma_{n}\right), z_{n} \in T\left(y_{n}, \gamma_{n}\right)$ such that $\left\{y_{n}\right\} \rightarrow y,\left\{z_{n}\right\} \rightarrow z$, for all $n$. As $x_{n} \in \Sigma_{s}^{\gamma_{0} \lambda_{0}}(\xi, \varepsilon)$ we have

$$
\left\langle Q\left(z_{n}\right), \eta\left(y_{n}, x_{n}, \lambda_{n}\right)\right\rangle+\varepsilon \nless 0 .
$$

By the continuity of $Q, \eta,\langle\cdot, \cdot\rangle$ and id, it follows that $\langle\cdot, \cdot\rangle+$ id is continuous. So we have

$$
\langle Q(z), \eta(y, x, \lambda)\rangle+\varepsilon \nless 0,
$$

and we see a contradiction. Hence $x \in \Sigma_{s}^{\gamma_{0} \lambda_{0}}(\xi, \varepsilon)$. Thus, $\Sigma_{s}^{\gamma_{0} \lambda_{0}}(\xi, \varepsilon)$ is closed.

Next, we provide sufficient conditions for the two sets to coincide.

Lemma 2.20 Assume for problem (MQVIP) the following conditions to hold:

(i) $K_{1}(x, \cdot)$ is closed at $\gamma_{0}$, and $K_{2}(x, \cdot)$ is lsc at $\gamma_{0}$;

(ii) in $K_{2}\left(K_{1}(A, \Gamma), \Gamma\right) \times\left\{\gamma_{0}\right\}, T$ is usc and compact-valued if $\alpha=\mathrm{w}$ (or $\left.\alpha=\mathrm{m}\right)$, and lsc if $\alpha=\mathrm{s}$.

Then $\Psi_{\alpha}\left(\gamma_{0}, \lambda_{0}\right)=\bigcap_{\varepsilon \in \operatorname{int} C, \xi>0} \Sigma_{\alpha}^{\gamma_{0} \lambda_{0}}(\xi, \varepsilon)$, for every $\left(\gamma_{0}, \lambda_{0}\right) \in \Gamma \times \Lambda$.

Proof We present only the proof for the case where $\alpha=\mathrm{s}$. We first prove that $\bigcap_{\varepsilon \in \operatorname{int} C} \Sigma_{s}^{\gamma_{0} \lambda_{0}}(\xi, \varepsilon)=\Omega_{s}\left(\gamma_{0}, \lambda_{0}, \varepsilon\right)$. It is easy to see that $\bigcap_{\varepsilon \in \operatorname{int} C} \Sigma_{s}^{\gamma_{0} \lambda_{0}}(\xi, \varepsilon) \supseteq \Omega_{s}\left(\gamma_{0}, \lambda_{0}, \varepsilon\right)$. Thus, we only need to show that $\bigcap_{\varepsilon \in \text { int } C} \Sigma_{s}^{\gamma_{0} \lambda_{0}}(\xi, \varepsilon) \subseteq \Omega_{s}\left(\gamma_{0}, \lambda_{0}, \varepsilon\right)$. Indeed, let $x \in$ $\bigcap_{\varepsilon \in \operatorname{int} C} \Sigma_{s}^{\gamma_{0} \lambda_{0}}(\xi, \varepsilon)$, there are $\gamma_{n} \in B\left(\gamma_{0}, \xi\right)$ and $\lambda_{n} \in B\left(\lambda_{0}, \xi\right)$ such that $\forall y \in K_{2}\left(x, \gamma_{n}\right)$, $\forall z \in T\left(y, \gamma_{n}\right)$ satisfying

$$
\left\langle Q(z), \eta\left(y, x, \lambda_{n}\right)\right\rangle+\varepsilon \nless 0 \text {. }
$$

Since $x \in K_{1}\left(x, \gamma_{n}\right), \gamma_{n} \rightarrow \gamma_{0}$ and $K_{1}$ is closed, we have $x \in K_{1}\left(x, \gamma_{0}\right)$. Now we verify that $x \in \Omega\left(\gamma_{0}, \lambda_{0}, \varepsilon\right)$. Indeed, for each $y \in K_{2}\left(x, \gamma_{0}\right)$, by the semicontinuity of $K_{2}(x, \cdot)$ at $\gamma_{0}$ and the semicontinuity of $T$ at $\left(y, \gamma_{0}\right)$, there exist $y_{n} \in K_{2}\left(x, \gamma_{n}\right)$ and $z_{n} \in T\left(y_{n}, \gamma_{n}\right)$ such that $\left\{y_{n}\right\} \rightarrow y,\left\{z_{n}\right\} \rightarrow z$. As $x \in \Omega_{s}\left(\gamma_{n}, \lambda_{n}, \varepsilon\right)$, we have

$$
\left\langle Q\left(z_{n}\right), \eta\left(y_{n}, x, \lambda_{n}\right)\right\rangle+\varepsilon \nless 0 .
$$


By the continuity of $Q, \eta,\langle\cdot, \cdot\rangle,\langle\cdot, \cdot\rangle+\mathrm{id}$, and $\left(y_{n}, z_{n}, \gamma_{n}, \lambda_{n}\right) \rightarrow\left(y, z, \gamma_{0}, \lambda_{0}\right)$. We have

$$
\left\langle Q(z), \eta\left(y, x, \lambda_{0}\right)\right\rangle+\varepsilon \nless 0,
$$

i.e.,

$$
\bigcap_{\varepsilon \in \operatorname{int} C} \Sigma_{s}^{\gamma_{0} \lambda_{0}}(\xi, \varepsilon) \subseteq \Omega_{s}\left(\gamma_{0}, \lambda_{0}, \varepsilon\right) .
$$

Hence

$$
\bigcap_{\varepsilon \in \operatorname{int} C} \Sigma_{s}^{\gamma_{0} \lambda_{0}}(\xi, \varepsilon)=\Omega_{s}\left(\gamma_{0}, \lambda_{0}, \varepsilon\right) .
$$

It is clear that

$$
\Psi_{s}\left(\gamma_{0}, \lambda_{0}\right)=\bigcap_{\xi>0} \Omega_{s}\left(\gamma_{0}, \lambda_{0}, \varepsilon\right)=\bigcap_{\xi>0, \varepsilon \in \operatorname{int} C} \Sigma_{s}^{\gamma_{0} \lambda_{0}}(\xi, \varepsilon) .
$$

The following theorem shows the well-posedness in the generalized sense at $\left(\gamma_{0}, \lambda_{0}\right)$ for (MQVIP) by using the Kuratowski measure $\zeta$.

Theorem 2.21 Let $X$ be complete, $\Gamma, \Lambda$ be finite dimensional and the following conditions hold:

(i) $K_{1}$ is closed in $A \times\left\{\gamma_{0}\right\}$, and in $K_{1}(A, \Gamma) \times\left\{\gamma_{0}\right\}, K_{2}$ is lsc;

(ii) in $K_{2}\left(K_{1}(A, \Gamma), \Gamma\right) \times\left\{\gamma_{0}\right\}$, $T$ is usc and compact-valued if $\alpha=\mathrm{w}($ or $\alpha=\mathrm{m})$, and lsc if $\alpha=\mathrm{s}$.

Then (MQVIP) is well-posed in the generalized sense at $\left(\gamma_{0}, \lambda_{0}\right)$ if and only if

$$
\Sigma_{\alpha}^{\gamma_{0} \lambda_{0}}(\xi, \varepsilon) \neq \emptyset, \quad \forall \xi>0, \varepsilon \in \operatorname{int} C \quad \text { and } \quad \zeta\left(\Sigma_{\alpha}^{\gamma_{0} \lambda_{0}}(\xi, \varepsilon)\right) \rightarrow 0 \quad \text { as }(\xi, \varepsilon) \rightarrow(0,0)
$$

Proof Similar arguments can be applied in the three cases. We present only the proof for the case where $\alpha=\mathrm{s}$. Now we suppose that (MQVIP) is well-posed in the generalized sense at $\left(\gamma_{0}, \lambda_{0}\right)$. Let $\Psi_{s}$ be a solution set of (MQVIP $\left.{ }^{\gamma \lambda}\right)$ for all $(\gamma, \lambda) \in \Gamma \times \Lambda$. Then, from Theorem 2.6, we see that $\Psi_{s}\left(\gamma_{0}, \lambda_{0}\right)$ is a nonempty compact. Clearly $\Psi_{s}\left(\gamma_{0}, \lambda_{0}\right) \subseteq \Sigma_{s}^{\gamma_{0} \lambda_{0}}(\xi, \varepsilon)$, $\forall \xi>0, \varepsilon \in \operatorname{int} C$. Now we show that

$$
\zeta\left(\Sigma_{s}^{\gamma_{0} \lambda_{0}}(\xi, \varepsilon)\right) \rightarrow 0 \quad \text { as }(\xi, \varepsilon) \rightarrow(0,0)
$$

Indeed, since $\Psi_{s}\left(\gamma_{0}, \lambda_{0}\right) \subseteq \Sigma_{s}^{\gamma_{0} \lambda_{0}}(\xi, \varepsilon), \forall \xi>0, \varepsilon \in$ int $C$. Using the concept of Hausdorff metric, we have

$$
\begin{aligned}
H & \left(\Sigma_{s}^{\gamma_{0} \lambda_{0}}(\xi, \varepsilon), \Psi_{s}\left(\gamma_{0}, \lambda_{0}\right)\right) \\
& =\max \left\{H^{*}\left(\Sigma_{s}^{\gamma_{0} \lambda_{0}}(\xi, \varepsilon), \Psi_{s}\left(\gamma_{0}, \lambda_{0}\right)\right), H^{*}\left(\Psi_{s}\left(\gamma_{0}, \lambda_{0}\right), \Sigma_{s}^{\gamma_{0} \lambda_{0}}(\xi, \varepsilon)\right)\right\} \\
& =H^{*}\left(\Sigma_{s}^{\gamma_{0} \lambda_{0}}(\xi, \varepsilon), \Psi_{s}\left(\gamma_{0}, \lambda_{0}\right)\right) .
\end{aligned}
$$

Suppose that $\Psi_{s}\left(\gamma_{0}, \lambda_{0}\right) \subseteq \bigcup_{i=1}^{n} L_{i}$, diam $L_{i}<\vartheta, i=1,2, \ldots, n$, for some $n \in \mathbb{N}$. 
We set $\Delta_{i}=\left\{t \in A \mid d\left(t, L_{i}\right) \leq H\left(\Sigma_{s}^{\gamma_{0} \lambda_{0}}(\xi, \varepsilon), \Psi_{s}\left(\gamma_{0}, \lambda_{0}\right)\right)\right\}$.

We claim that $\Sigma_{s}^{\gamma_{0} \lambda_{0}}(\xi, \varepsilon) \subseteq \bigcup_{i=1}^{n} \Delta_{i}$. Indeed, let $x \in \Sigma_{s}^{\gamma_{0} \lambda_{0}}(\xi, \varepsilon)$. Then $d\left(x, \Psi_{s}\left(\gamma_{0}, \lambda_{0}\right)\right) \leq$ $H\left(\Sigma_{s}^{\gamma_{0} \lambda_{0}}(\xi, \varepsilon), \Psi_{s}\left(\gamma_{0}, \lambda_{0}\right)\right)$. Since $\Psi_{s}\left(\gamma_{0}, \lambda_{0}\right) \subseteq \bigcup_{i=1}^{n} L_{i}$, we see that

$$
d\left(x, \bigcup_{i=1}^{n} L_{i}\right) \leq H\left(\Sigma_{s}^{\gamma_{0} \lambda_{0}}(\xi, \varepsilon), \Psi_{s}\left(\gamma_{0}, \lambda_{0}\right)\right) .
$$

Hence, there is $k$ such that $d\left(x, L_{k}\right) \leq H\left(\Sigma_{s}^{\gamma_{0} \lambda_{0}}(\xi, \varepsilon), \Psi_{s}\left(\gamma_{0}, \lambda_{0}\right)\right)$, i.e., $x \in \Delta_{k}$. So

$$
\Sigma_{s}^{\gamma_{0} \lambda_{0}}(\xi, \varepsilon) \subseteq \bigcup_{i=1}^{n} \Delta_{i}
$$

Note further that

$$
\begin{aligned}
\operatorname{diam} \Delta_{i} & =\operatorname{diam} L_{i}+2 H\left(\Sigma_{s}^{\gamma_{0} \lambda_{0}}(\xi, \varepsilon), \Psi_{s}\left(\gamma_{0}, \lambda_{0}\right)\right) \\
& \leq \vartheta+2 H\left(\Sigma_{s}^{\gamma_{0} \lambda_{0}}(\xi, \varepsilon), \Psi_{s}\left(\gamma_{0}, \lambda_{0}\right)\right) .
\end{aligned}
$$

Hence,

$$
\zeta\left(\Sigma_{s}^{\gamma_{0} \lambda_{0}}(\xi, \varepsilon)\right) \leq 2 H\left(\Sigma_{s}^{\gamma_{0} \lambda_{0}}(\xi, \varepsilon), \Psi_{s}\left(\gamma_{0}, \lambda_{0}\right)\right)+\zeta\left(\Psi_{s}\left(\gamma_{0}, \lambda_{0}\right)\right) .
$$

Since $\Psi_{s}\left(\gamma_{0}, \lambda_{0}\right)$ is compact, $\zeta\left(\Psi_{s}\left(\gamma_{0}, \lambda_{0}\right)\right)=0$, so we have

$$
\zeta\left(\Sigma_{s}^{\gamma_{0} \lambda_{0}}(\xi, \varepsilon)\right) \leq 2 H^{*}\left(\Sigma_{s}^{\gamma_{0} \lambda_{0}}(\xi, \varepsilon), \Psi_{s}\left(\gamma_{0}, \lambda_{0}\right)\right) .
$$

Now we prove that

$$
H^{*}\left(\Sigma_{s}^{\gamma_{0} \lambda_{0}}(\xi, \varepsilon)\right) \rightarrow 0 \quad \text { as }(\xi, \varepsilon) \rightarrow(0,0) .
$$

Suppose to the contrary that

$$
H^{*}\left(\Sigma_{s}^{\gamma_{0} \lambda_{0}}(\xi, \varepsilon)\right) \nrightarrow 0 \quad \text { as }(\xi, \varepsilon) \rightarrow(0,0) .
$$

There are $\theta>0,\left(\xi_{n}, \varepsilon_{n}\right) \rightarrow(0,0)$, and $x_{n} \in \Sigma_{s}^{\gamma_{0} \lambda_{0}}\left(\xi_{n}, \varepsilon_{n}\right)$ such that

$$
d\left(x_{n}, \Psi_{s}\left(\gamma_{0}, \lambda_{0}\right)\right) \geq \theta>0, \quad \forall n \in \mathbb{N} .
$$

$\left\{x_{n}\right\}$ is an approximating sequence of (MQVIP). By the well-posedness in the generalized sense of (MQVIP) at $\left(\gamma_{0}, \lambda_{0}\right)$, there is a subsequence $\left\{x_{k}\right\}$ of $\left\{x_{n}\right\}$ converging to some point of $\Psi_{s}\left(\gamma_{0}, \lambda_{0}\right)$, which is impossible as $d\left(x_{n}, \Psi_{s}\left(\gamma_{0}, \lambda_{0}\right)\right) \geq \theta>0, \forall n \in \mathbb{N}$. Hence

$$
\zeta\left(\Sigma_{s}^{\gamma_{0} \lambda_{0}}(\xi, \varepsilon)\right) \rightarrow 0 \quad \text { as }(\xi, \varepsilon) \rightarrow(0,0)
$$

Conversely, $\zeta\left(\Sigma_{s}^{\gamma_{0} \lambda_{0}}(\xi, \varepsilon)\right) \rightarrow 0$ as $(\xi, \varepsilon) \rightarrow(0,0)$. By Lemma 2.19 , we see that $\Sigma_{s}^{\gamma_{0} \lambda_{0}}(\xi, \varepsilon)$ is closed, for all $\xi>0, \varepsilon \in$ int $C$. By Lemma 2.20, we have

$$
\Psi_{s}\left(\gamma_{0}, \lambda_{0}\right)=\bigcap_{\varepsilon \in \operatorname{int} C, \xi>0} \Sigma_{s}^{\gamma_{0} \lambda_{0}}(\xi, \varepsilon) .
$$


Since $\zeta\left(\Sigma_{s}^{\gamma_{0} \lambda_{0}}(\xi, \varepsilon)\right) \rightarrow 0$ as $(\xi, \varepsilon) \rightarrow(0,0)$, the regular measure properties of $\zeta$ imply that $\Psi_{s}\left(\gamma_{0}, \lambda_{0}\right)$ is compact and

$$
H\left(\Sigma_{s}^{\gamma_{0} \lambda_{0}}(\xi, \varepsilon), \Psi_{s}\left(\gamma_{0}, \lambda_{0}\right)\right) \rightarrow 0 \quad \text { as }(\xi, \varepsilon) \rightarrow(0,0)
$$

Let $\left\{x_{n}\right\}$ be an approximating sequence for (MQVIP) corresponding to $\left\{\left(\gamma_{n}, \lambda_{n}\right)\right\}$, where $\left\{\gamma_{n}\right\} \rightarrow \gamma_{0}$ and $\left\{\lambda_{n}\right\} \rightarrow \lambda_{0}$. There is $\left\{\varepsilon_{n}\right\} \rightarrow 0$ such that $\forall y \in K_{2}\left(x_{n}, \gamma_{n}\right), \forall z \in T\left(y, \gamma_{n}\right)$ satisfying

$$
\left\langle Q(z), \eta\left(y, x_{n}, \lambda_{n}\right)\right\rangle+\varepsilon_{n} \nless 0, \quad \forall n \in \mathbb{N} .
$$

This means that $x_{n} \in \Sigma_{s}^{\gamma_{0} \lambda_{0}}\left(\xi_{n}, \varepsilon_{n}\right)$ with $\xi_{n}:=\max \left\{d\left(\gamma_{0}, \gamma_{n}\right), d\left(\lambda_{0}, \lambda_{n}\right)\right\}$. We see that

$$
d\left(x_{n}, \Psi_{s}\left(\gamma_{0}, \lambda_{0}\right)\right) \leq H\left(\Sigma_{s}^{\gamma_{0} \lambda_{0}}\left(\xi_{n}, \varepsilon_{n}\right), \Psi_{s}\left(\gamma_{0}, \lambda_{0}\right)\right) \rightarrow 0 \quad \text { as } n \rightarrow+\infty
$$

Hence, there is $\bar{x}_{n} \in \Psi_{s}\left(\gamma_{0}, \lambda_{0}\right)$ such that

$$
d\left(x_{n}, \bar{x}_{n}\right) \rightarrow 0 \quad \text { as } n \rightarrow+\infty .
$$

By the compactness of $\Psi_{s}\left(\gamma_{0}, \lambda_{0}\right)$, there is a subsequence $\left\{\bar{x}_{n_{k}}\right\}$ of $\left\{\bar{x}_{n}\right\}$ convergent to some point $x_{0}$ of $\Psi_{s}\left(\gamma_{0}, \lambda_{0}\right)$. Therefore, the corresponding subsequence $\left\{x_{n_{k}}\right\}$ of $\left\{x_{n}\right\}$ tends to $x_{0}$. Hence, (MQVIP) is well-posed in the generalized sense at $\left(\gamma_{0}, \lambda_{0}\right)$.

Remark 2.22 In cases as in Remark 2.4(i), Theorems 3.3, 3.4, and 3.5-3.6 in [22] are particular cases of Theorems 2.13, 2.21, and 2.9, respectively. However, the assumptions and our proof methods are very different from Theorems 3.3, 3.4, and 3.5-3.6 in [22].

The following example shows that the closedness of $K_{1}$ in Theorem 2.21 cannot be dropped.

Example 2.23 Let $X=Y=\mathbb{R}, A=B=[-5,5], \Gamma=\Lambda=[0,1], C=\mathbb{R}_{+}, \gamma_{0}=0, H$ be an identity map, and let $K_{1}, K_{2}: A \times \Gamma \rightarrow 2^{A}, T: A \times \Gamma \rightarrow 2^{L(X, Y)}$, and $\eta: A \times A \times \Gamma \rightarrow A$ be defined by

$$
\begin{aligned}
& K_{1}(x, \gamma)=[-5 \gamma, 5], \\
& \eta(y, x, \gamma)=x(x-y), \\
& T(y, \gamma)=\{1\}, \\
& K_{2}(x, \gamma)=[0,5] .
\end{aligned}
$$

We show that $K_{2}$ is lsc in $K_{1}(A, \Gamma) \times \Gamma$ and the condition (ii) of Theorem 2.21 is easily seen to be fulfilled and $\Sigma_{\alpha}^{\gamma_{0} \lambda_{0}}(\xi, \varepsilon) \subseteq[-5,5]$. Hence, $\zeta\left(\Sigma_{\alpha}^{\gamma_{0} \lambda_{0}}(\xi, \varepsilon)\right) \rightarrow 0$ as $(\xi, \varepsilon) \rightarrow(0,0)$. But the family $\left\{\left(\operatorname{MQVIP}^{\gamma \lambda}\right):(\gamma, \lambda) \in \Gamma \times \Lambda\right\}$ is not well-posed in the generalized sense at $(0,0)$. The reason is that $K_{1}$ is not closed at $(A, 0)$. Indeed, we let $\gamma_{n}=x_{n}=\frac{1}{n} \rightarrow 0$, as $n \rightarrow \infty$ and $t_{n}=\frac{1}{n} \in K_{1}\left(x_{n}, \gamma_{n}\right)=\left(-\frac{5}{n}, 5\right], \forall n \in \mathbb{N}$. It is clear that $\left\{t_{n}\right\}$ is convergent to $0 \notin K_{1}(0,0)=(0,5]$. In fact, $\Omega_{\alpha}\left(\gamma_{0}, \lambda_{0}, \varepsilon\right)=\Sigma_{\alpha}^{\gamma_{0} \lambda_{0}}(\xi, \varepsilon)=\{5\}$. 
The following example shows that the lower semicontinuity of $K_{2}$ in Theorem 2.21 is essential.

Example 2.24 Let $X=Y=\mathbb{R}, A=B=[-2,2], \Gamma=\Lambda=[0,1], C=\mathbb{R}_{+}, \varepsilon \in \operatorname{int} C, \xi>0$, $\gamma_{0}=0, H$ be an identity map, and let $K_{1}, K_{2}: A \times \Gamma \rightarrow 2^{A}, T: A \times \Gamma \rightarrow 2^{L(X, Y)}$, and $\eta$ : $A \times A \times \Gamma \rightarrow A$ be defined by

$$
\begin{aligned}
& K_{1}(x, \gamma)=[0,2], \\
& \eta(y, x, \gamma)=x+y, \\
& T(y, \gamma)=\{1\}, \\
& K_{2}(x, \gamma)= \begin{cases}\{-2,0,2\}, & \text { if } \gamma=0, \\
\{0,2\}, & \text { otherwise. }\end{cases}
\end{aligned}
$$

We show that $K_{2}$ is lsc in $K_{1}(A, \Gamma) \times \Gamma$ and the condition (ii) of Theorem 2.21 is easily seen to be fulfilled and $\Sigma_{\alpha}^{\gamma_{0} \lambda_{0}}(\xi, \varepsilon) \subseteq[-2,2]$. Hence, $\zeta\left(\Sigma_{\alpha}^{\gamma_{0} \lambda_{0}}(\xi, \varepsilon)\right) \rightarrow 0$ as $(\xi, \varepsilon) \rightarrow(0,0)$. But the family $\left\{\left(\right.\right.$ MQVIP $\left.\left.^{\gamma \lambda}\right):(\gamma, \lambda) \in \Gamma \times \Lambda\right\}$ is not well-posed in the generalized sense at $(0,0)$. The reason is that $K_{2}$ is not lower semicontinuous. In fact

$$
\Omega_{\alpha}(\gamma, \lambda, \varepsilon)=\Sigma_{\alpha}^{\gamma \lambda}(\xi, \varepsilon)= \begin{cases}{[2-\varepsilon, 2] \cap[0,2],} & \text { if } \gamma=0, \\ {[0,2],} & \text { if } \gamma \in(0,1] .\end{cases}
$$

The following example shows that all assumptions of Theorem 2.21 are fulfilled.

Example 2.25 Let $X=Y=\mathbb{R}, A=B=\Gamma=\Lambda=[0,2], C=\mathbb{R}_{+}, \varepsilon \in \operatorname{int} C, \xi>0, \gamma_{0}=0, H$ be an identity map, and let $K_{1}, K_{2}: A \times \Gamma \rightarrow 2^{A}, T: A \times \Gamma \rightarrow 2^{L(X, Y)}$, and $\eta: A \times A \times \Gamma \rightarrow A$ be defined by

$$
\begin{aligned}
& K_{1}(x, \gamma)=K_{2}(x, \gamma)=[\gamma, \gamma+2], \\
& \eta(y, x, \gamma)=\left\{2^{\gamma^{2}+1}-\varepsilon\right\}, \\
& T(y, \gamma)=\left\{\frac{1}{2^{\gamma^{2}+1}}\right\} .
\end{aligned}
$$

We show that the assumptions (i) and (ii) of Theorem 2.21 are easily seen to be fulfilled and

$$
\Omega_{\alpha}(\gamma, \lambda, \varepsilon)= \begin{cases}{[\gamma, \gamma+2],} & \text { if } \gamma \in(0,1], \\ {[0,2],} & \text { if } \gamma=0,\end{cases}
$$

and $\Sigma_{\alpha}^{\gamma_{0} \lambda_{0}}(\xi, \varepsilon) \subseteq[0,2]$. Hence, $\zeta\left(\Sigma_{\alpha}^{\gamma_{0} \lambda_{0}}(\xi, \varepsilon)\right) \rightarrow 0$ as $(\xi, \varepsilon) \rightarrow(0,0)$, and the family $\left\{\left(\operatorname{MQVIP}^{\gamma \lambda}\right):(\gamma, \lambda) \in \Gamma \times \Lambda\right\}$ is well-posed in the generalized sense at $(0,0)$. 


\section{Acknowledgements}

The author is grateful to Prof. Phan Quoc Khanh and Prof. Lam Quoc Anh for their help in the research process. The author also thanks the three anonymous referees for their valuable remarks and suggestions, which helped them to improve considerably the article.

\section{Received: 13 August 2013 Accepted: 25 April 2014 Published: 12 May 2014}

\section{References}

1. Giannessi, F: Theorems of alternative, quadratic programmes and complementarity problems. In: Cottle, RW, Giannessi, F, Lions, JL (eds.) Variational Inequalities and Complementarity Problems, pp. 151-186. Wiley, Chichester (1980)

2. Chen, CR, Li, SJ: Upper Hölder estimates of solutions to parametric primal and dual vector quasi-equilibria. J. Ind. Manag. Optim. 8,691-703 (2012)

3. Lalitha, CS, Bhatia, G: Stability of parametric quasivariational inequality of the Minty type. J. Optim. Theory Appl. 148 281-300 (2011)

4. Hung, NV: Stability of solution set for parametric generalized vector mixed quasivariational inequality problem. J. Inequal. Appl. 2013, 176 (2013)

5. Hung, NV: Existence conditions for symmetric generalized quasi-variational inclusion problems. J. Inequal. Appl. 2013, 40 (2013)

6. Li, SJ, Chen, CR: Stability of weak vector variational inequality. Nonlinear Anal. TMA 70, 1528-1535 (2009)

7. Morgan, J, Scalzo, V: Pseudocontinuity in optimization and nonzero sum games. J. Optim. Theory Appl. 120, 181-197 (2004)

8. Tykhonov, AN: On the stability of the functional optimization problem. USSR Comput. Math. Math. Phys. 6, 28-33 (1966)

9. Zolezzi, T: Condition number theorems in optimization. SIAM J. Optim. 14, 507-516 (2003)

10. Anh, LQ, Khanh, PQ: On the stability of the solution sets of general multivalued vector quasiequilibrium problems. J. Optim. Theory Appl. 135, 271-284 (2007)

11. Long, $\mathrm{XJ}$, Huang, $\mathrm{NJ}$, Teo, KL: Existence and stability of solutions for generalized strong vector quasi-equilibrium problems. Math. Comput. Model. 47, 445-451 (2008)

12. Hung, NV: Continuity of solutions for parametric generalized quasivariational relation problems. Fixed Point Theory Appl. 2012, 102 (2012)

13. Hung, NV: Sensitivity analysis for generalized quasi-variational relation problems in locally G-convex spaces. Fixed Point Theory Appl. 2012, 158 (2012)

14. Levitin, ES, Polyak, BT: Convergence of minimizing sequences in conditional extremum problem. Sov. Math. Dokl. 7, 764-767 (1966)

15. Lignola, MB, Morgan, J: Well-posedness for optimization problems with constraints defined by variational inequalities having a unique solution. J. Glob. Optim. 16, 57-67 (2000)

16. Morgan, J, Scalzo, V: Discontinuous but well-posed optimization problems. SIAM J. Optim. 17, 861-870 (2006)

17. Zolezzi, T: Well-posedness criteria in optimization with applications to the calculus of variations. Nonlinear Anal. TMA $25,437-453(1995)$

18. Zolezzi, T: Well-posedness and optimization under perturbations. Ann. Oper. Res. 101, 351-361 (2001)

19. Zolezzi, T: On well-posedness and conditioning in optimization. Z. Angew. Math. Mech. 84, 435-443 (2004)

20. Revalski, JP: Hadamard and strong well-posedness for convex programs. SIAM J. Optim. 7, 519-526 (1997)

21. Fang, YP, Hu, R: Parametric well-posedness for variational inequalities, defined by bifunctions. Comput. Math. Appl. 53, 1306-1316 (2007)

22. Fang, YP, Huang, NJ: Well-posedness for vector variational inequality and constrained vector optimization. Taiwan. J. Math. 11, 1287-1300 (2007)

23. Lalitha, CS, Bhatia, G: Levitin-Polyak well-posedness for parametric qusivariational inequality problem of the Minty type. Positivity 16, 527-541 (2012)

24. Anh, LQ, Khanh, PQ, Van, DTM, Yao, JC: Well-posedness for vector quasiequilibria. Taiwan. J. Math. 13, 713-737 (2009)

25. Anh, LQ, Khanh, PQ, Van, DTM: Well-posedness without semicontinuity for parametric quasiequilibria and quasioptimization. Comput. Math. Appl. 62, 2045-2057 (2011)

26. Fang, YP, Hu, R, Huang, NJ: Well-posedness for equilibrium problems and for optimization problems with equilibrium constraints. Comput. Math. Appl. 55, 89-100 (2008)

27. Huang, NJ, Long, XJ, Zhao, CW: Well-posedness for vector quasiequilibrium problems with applications. J. Ind. Manag. Optim. 5, 341-349 (2009)

28. Kimura, K, Liou, YC, Wu, SY, Yao, JC: Well-posedness for parametric vector equilibrium problems with applications. J. Ind. Manag. Optim. 4, 313-327 (2008)

29. Li, SJ, Li, MH: Levitin-Polyak well-posedness of vector equilibrium problems. Math. Methods Oper. Res. 69, 125-140 (2008)

30. Li, QY, Wang, SH: Well-posedness for parametric strong vector quasi-equilibrium problems with applications. Fixed Point Theory Appl. 2011, 62 (2011)

31. Long, XJ, Huang, NJ: Metric characterizations of $\alpha$-well-posedness for symmetric quasi-equilibrium problems. J. Glob. Optim. 45, 459-471 (2009)

32. Long, XJ, Huang, NJ, Teo, KL: Levitin-Polyak well-posedness for equilibrium problems with functional constraints. J. Inequal. Appl. 2008, Article ID 657329 (2008)

33. Peng, JW, Wu, SY: The generalized Tykhonov well-posedness for system of vector quasi-equilibrium problems. Optim. Lett. 4, 501-512 (2010)

34. Aubin, JP, Ekeland, I: Applied Nonlinear Analysis. Wiley, New York (1984)

35. Berge, C: Topological Spaces. Oliver \& Boyd, London (1963)

36. Aubin, JP, Frankowska, H: Set-Valued Analysis. Birkhäuser Boston, Boston (1990)

37. Kuratowski, K: Topology, vol. 1. Academic Press, New York (1966) 
38. Kuratowski, K: Topology, vol. 2. Academic Press, New York (1968)

39. Banas, J, Goebel, K: Measures of Noncompactness in Banach Spaces. Lecture Notes in Pure and Applied Mathematics, vol. 60. Dekker, New York (1980)

40. Rakočević, V: Measures of noncompactness and some applications. Filomat 12, 87-120 (1998)

10.1186/1029-242X-2014-178

Cite this article as: Hung: Well-posedness for parametric generalized vector quasivariational inequality problems of the Minty type. Journal of Inequalities and Applications 2014, 2014:178

Submit your manuscript to a SpringerOpen ${ }^{\circ}$ journal and benefit from:

- Convenient online submission

Rigorous peer review

- Immediate publication on acceptance

- Open access: articles freely available online

- High visibility within the field

- Retaining the copyright to your article

Submit your next manuscript at $>$ springeropen.com 